\title{
Prime Decomposition: Understanding Uniqueness
}

\author{
Rina Zazkis and Stephen Campbell \\ Simon Fraser University
}

\begin{abstract}
This study investigates procedural and conceptual aspects in preservice elementary school teachers' understanding of the Fundamental Theorem of Arithmetic. The data were collected by the means of a written questionnaire and individual interviews. The results suggest that the idea of the uniqueness of prime decomposition is very difficult to grasp. Participants' responses indicated, either implicitly or explicitly, that a possibility of alternative prime decompositions was often not overruled. and this influenced students' ability to make inferences regarding factors and divisors of natural numbers. Some pedagogical implications are discussed.
\end{abstract}

Andy was asked whether $17^{3}$ was a square number. After calculating $17^{3}$ and observing that its square root was not an integer, Andy gave a negative answer. Patty was asked whether 11 was a divisor of $M$, where $M=3^{3} \times 5^{2} \times 7$. After calculating the number $M$ and dividing the product by 11 , Patty reached her negative conclusion. Bob was asked whether $K$ could have 13 as a divisor, where $K=16,199=97 \times 167$ and 97,167 are both primes. He claimed it was quite possible because $K$ ended with 9 , but had actually to perform division to infer that $K$ was not divisible by 13 .

Each one of these questions presents a number in its prime decomposition and invites a consideration of its divisors. A common feature in each one of the responses forms the focus of this article. Each response appears to admit the possibility of an alternative prime decomposition and, hence, reveals an inadequate understanding of one of the basic ideas of Number Theory-The Fundamental Theorem of Arithmetic.

The Fundamental Theorem of Arithmetic claims that decomposition of a composite number into its prime factors exists and is unique except for the order in which the prime factors appear in the product. This theorem is a part of the core mathematics curriculum for preservice elementary teachers in many institutions, even though the formal mathematical proof of the theorem is usually not provided for this audience. On several occasions we have noticed that many

This study was supported in part by grant \#410-93-1129 from the Social Sciences and Humanities Research Council of Canada.

Correspondence and requests for reprints should be sent to Rina Zazkis, Faculty of Education, Simon Fraser University, Burnaby, B.C. V5A 156 Canada. 
preservice elementary school teachers are familiar with the theorem, may articulate and explain its meaning, but fail to apply it in various problem-solving situations. The purpose of our study was to investigate this specific phenomenon in order to contribute to an improved pedagogical understanding of the construction of knowledge of natural numbers and their multiplicative structure. Following Freudenthal (1983), we use the term multiplicative structure to refer to "the whole of the relations (of all possible products) . . . and all one can think about in this context" (pp. 112-113). In particular, we will be thinking about products pertaining to the decomposition of natural numbers into their prime factors.

This study is a part of the ongoing research on teachers' content knowledge in mathematics. The specific focus of our inquiry is on teachers' understanding of topics in elementary Number Theory. In previous research we have investigated topics of divisibility and factorization (Zazkis \& Campbell, 1994, 1996). Extending our study to topics involving the Fundamental Theorem of Arithmetic is not an isolated goal in and of itself, but rather a natural progression of our investigations in this area.

\section{CONCEPTUAL FRAMEWORK}

Several overlapping ideas about the development of mathematical knowledge and its nature form the conceptual framework for this study. A repeating theme in the literature is that of the formation of cognitive structures (Piaget, 1972) or conceptual entities (Greeno, 1983). More recently, such conceptual entities have been addressed in terms of mathematical or mental objects and similar, though not identical, ideas regarding object construction have been proposed by various authors (Artigue, 1992; Douady, 1985; Dubinsky, 1991; Dubinsky, I.eron, Dautemann, \& Zazkis, 1994; Sfard, 1991). A common theme highlights the importance of object construction, referred to as encapsulation (Dubinsky) or reification (Sfard), in mathematical understanding. These terms theoretically express the transition from procedural knowledge to conceptual understanding. Furthermore, emphasis has been made on how the treatment of mathematical entities as cognitive objects not only contributes to the mathematical understanding of the learner, but also leads to progress in the historical development of mathematical ideas in general. These researchers seem to agree that there is a difficulty for the learner to attain object construction and that specific instructional treatments may help overcome this difficulty.

\section{METHOD}

The participants in our research were preservice elementary school teachers enrolled in a course "Foundations of Mathematics for Teachers," which served as a prerequisite course for teacher certification. The data acquired are based on written responses by 54 students on three assessment questions and subsequent 
clinical interviews with a group of volunteers from the same class. Both the questionnaire and the interviews were conducted after the topics of elementary number theory were covered in the course. This included topics such as prime and composite numbers, factor trees, prime decomposition and Fundamental Theorem of Arithmetic, divisibility and divisibility rules, greatest common divisor, and lowest common multiple, among others.

The three assessment questions were administered on three different occasions within a 2-week period of time and the participants were not temporally constrained in addressing them. In these questions, the students were invited to:

1. Consider the number $M=3^{3} \times 5^{2} \times 7$ and decide whether it is divisible by each of the numbers $7,5,3,2,15,11,9$, and 63 .

2. Consider the number $K=16,199=97 \times 67$ (where 97 and 167 are given as prime numbers) and decide whether $K$ may be divisible by $3,5,11,13$, and 17.

3. Look at the list of 15 numbers, such as $8^{2}, 17^{2}, 17^{3}, 234^{3}, 234^{6}, 5^{2} \times 17^{2}$, $5^{3} \times 7^{2}, 5^{6} \times 17^{2}, p^{3}$ where $p$ is prime, $C^{3}$ where $C$ is composite, and decide which numbers on the list are, or could be, perfect squares.

In the second question. the participants were explicitly asked to try and answer the question without calculators or paper and pencil calculations. In the first and the third questions, there were no specific instructions given regarding the strategy for solution. Explanations were requested for all the questions.

Qualitative data were obtained from 21 interviews, as part of a larger study investigating understandings of concepts from introductory number theory such as factors, multiples, and divisibility. The authors followed up in depth on participants' responses and focused on justifications for their decisions. During the interviews, each averaging about 1 hour, participants were prompted where appropriate for understanding that might not have becn apparent from their initial response. The interviews were audiotaped and then transcribed. The data for this report are drawn from the two following question sets:

\section{Question Set I}

Consider the number $M=3^{3} \times 5^{2} \times 7$. Is $M$ divisible by 7 ? Please explain.

Is $M$ divisible by $5,2,9,63,11,15$ ? Please explain.

\section{Question Set 2}

Is 391 divisible by 23 ? Please explain.

Is 391 divisible by 46 ? Please explain.

These questions were originally designed to investigate students' understanding of concepts associated with divisibility. In the course of this study, the theme of prime decomposition emerged as a coherent topic of analysis in its own right. Here, these data are analyzed in terms of students' procedural and conceptual 
understandings of prime decomposition in accordance with the Fundamental Theorem of Arithmetic.

\section{RESULTS AND INTERPRETATIONS}

Our participants indeed acquired knowledge of the Fundamental Theorem of Arithmetic: They could quote it when asked and illustrate their knowledge by, for example, decomposing a "big" number like 360 via different routes and ending with the same (unordered) list of prime factors. On the other hand, the examples of successful application of the Fundamental Theorem of Arithmetic were limited.

\section{Results From Written Questionnaire}

On Question 1, about one half ( 25 out of 54) of the students preferred to calculate $M$ and then check for divisibility by either performing division or applying specific divisibility rules. A majority (29 out of 54) of the participants were able to accompany their positive answer regarding the divisibility of $M$ by 7,5 , and 3 with a mathematical justification "since those are among the factors in prime decomposition."

On the other hand, 16 out of these 29 participants were unable to apply similar reasoning regarding divisibility by 2 and 11 ; that is, to claim that those primes were not among the factors in prime decomposition of $M$. Noting the "oddness" of $M$ resolved the problem of divisibility by 2 . When considering 11, they regressed to procedural calculations and only after carrying out division inferred that 11 was not among $M$ 's divisors. Participants' responses suggest that, for these 16 students a possibility of the existence of another prime decomposition was not overruled. It is likely that this possibility was not an explicit belief but rather a manifestation of a lack of understanding of the relationship between factors and divisors.

In Question 2, 15 out of 54 students claimed, applying specific divisibility rules, that 16,199 was not divisible by 5 or 3 , but may be divisible by 11,13 , or 17. For many students, the only way to find out (divisibility by 11, 13, or 17) was to perform division. Even though the number 16,199 was presented to students in its prime decomposition $(97 \times 167)$, it seemed, again, that at least implicitly our participants believed that an alternative prime decomposition might be possible. Lesley's comment on her questionnaire exemplifies an explicit case in this regard: "Because a number is divisible by two primes does not mean that it is not divisible by other primes."

Thirty-nine (out of 54) students claimed that 16,199 was not divisible by any of the numbers on the list; that is, $3,5,11,13$, and 17 . The arguments to justify this claim varied. Only 13 students used the idea of uniqueness of prime decomposition, either explicitly (e.g., "the prime decomposition of 16,199 is $167 \times 97$ and these are the only two prime divisors"), or implicitly (e.g., "for any of the 
following primes: $3,5,11,13$, or 17 -to be a factor of 16,199 , they would have to be part of its prime decomposition"). On three worksheets, explicit long division was carried out as calculators were not allowed on this question. Five students made the claim without providing any additional justification. Three students accompanied their correct decision with arguments, such as: "These numbers will not divide 16,199 , because 16,199 is a product of two prime numbers, forming a prime number itself." These explanations suggest that the concept of primeness was not understood.

The remaining 15 participants (out of 39 answering correctly) claimed that 16.199 cannot be divisible by $3,5,11,13$, and 17 because " 167 and 97 are both primes and cannot be decomposed any more." That is, the focus of the claim was not on $3,5,11,13$, and 17 being nonfactors of 16,199 , but on $3,5,11,13$, and 17 being nonfactors of 167 and 97 . The students making this claim were likely to have been thinking of prime decomposition as a factoring process, rather than conceptually in terms of a number expressed as a product of primes. We concur with Sfard $(1991,1992)$ that the step toward higher level object constructions seems to be particularly difficult. For these students, as a case in point, this step was not attained.

It is interesting to note that 5 out of these 15 participants wanted to overemphasize their answer by providing additional arguments, unnecessary for this case, for indivisibility of 16,199 by 3 and 5 , using conventional divisibility rules. Several students indicated what they thought (hoped, believed, guessed) the correct answer was, but would have preferred to check by performing division. It seemed as though they did not have much faith in their own argument, and wished to strengthen it. This finding appears consistent with Martin and Harel (1989) and Fischbein and Kedem (1982), who observed that many students who were convinced by deductive arguments wanted further empirical verification.

On the third question it seemed necessary for one third of the students (18 out of 54) to calculate the value of a number such as $17^{3}$ or $17^{6}$, and then to find its square root, in order to infer whether it was a perfect square. A majority of students who claimed that $17^{3}$ was not a square number explained their decision by observing, "It was cubed." The cubic form of the number was emphasized rather than its prime decomposition. Our conjecture is, even though we have not used this specific example, that similar reasoning could be applied by some participants claiming that $16^{3}$ was not a square number as well, because, "It was cubed." Some students recognized only the numbers of the form $B^{2}$, although not $B^{6}$ and not even $B^{2} A^{2}$, as perfect squares. According to Harel and Kaput (1991), "notations can act as substitutes for conceptual entities, supplanting the need for them" (p. 93). We believe that here the notation $B^{2}$ served as a substitute for a conceptual entity of a square number.

We turn now to the following two parts of Question 3: Is $p^{3}$ a square number, when $p$ is prime and is $C^{3}$ a square number, where $C$ is composite? Out of 54 students, only 5 treated the numbers $p^{3}$ and $C^{3}$ differently. Two students correctly 
claimed that $p^{3}$ was not a square, but $C^{3}$ could be a square. The other three students claimed that $p^{3}$ was not a square, but chose not to answer at all the same question about $C$. Because the time to respond was not limited, we take the lack of the answer as students' hesitation to decide.

The remaining 49 out of 54 students responded in exactly the same way to the first question as they did for the second, making no discernible distinction between prime and composite numbers. Two students claimed that in both cases they needed to know the values of $p$ and $C$ to make up their minds. The remaining 47 students claimed that both $p^{3}$ and $C^{3}$ were not square numbers. Similarity in explanations demonstrates that students were treating $C$ as one entity and not taking into account the prime decomposition of $C$. Inability to distinguish between the two cases by the overwhelming majority of students suggests that the students' constructions of concepts of prime and composite numbers require further refinement.

\section{Results From the Interviews}

In the interviews, as students engaged in various problems regarding divisibility, we found additional indications of gaps in students' understandings of the uniqueness of prime decomposition.

In the first question of the interview, participants were asked to consider the number $M=3^{3} \times 5^{2} \times 7$ and decide whether it was divisible by each of the numbers $7,5,3,2,15,11,9$, and 63 . Asking participants to consider the number given in its prime decomposition, we hoped to divert their attention from procedures when determining divisibility and motivate a focus toward the multiplicative structure of $M$. The numbers chosen in this question included prime and composite divisors of $M$ and prime nondivisors of $M$. Students' responses to prime versus composite divisors have been discussed in detail in Zazkis and Campbell (1996). Here we focus on students' different approaches in making inferences about factors and nonfactors. Consistent with the results of the written questionnaire, the "proof" or verification of divisibility was in most cases more readily achieved than the refutation. Patty, for example, noted that both 7 and 5 as factors were both divisors of $M\left(M-3^{3} \times 5^{2} \times 7\right)$, but then regressed to procedural calculations when asked about divisibility by 2 and 11 .

Interviewer: Okay. And will it $(M)$ be divisible by 2 ?

Patty: I would multiply each one and find out what the total number is. So $3 \times 3$ is $9 \times 3$ is 27 , and this 25 is $\times 7$, (pause) it's not, 2 doesn't go into it evenly.

Interviewer: So you computed the number and you got 4,725 , and now you are sure that it is not divisible by 2 .

Patty: Right.

Interviewer: But you were able to conclude about divisibility by 7 before you knew what was the number... 
Patty: Um hm.

Interviewer: So how is it?

Patty: $\quad$ Because 7 is a factor of it, so it's, what is it, the commutative law or associate law -7 is a factor of it . . .

Interviewer: And what about divisibility of $M$ by 11 ?

Patty: I would divide 4,725 by 11 to find out.

It appears that Patty may have been thinking that 2 and 11 could possibly be divisors of $M$ even if they are not actually factors of $M$. For some participants, the question about 2 seemed easier than the question about 11 , when they noted that, " $M$ is an odd number" (as a product of odd numbers), so " 2 can't go into it." For them, the mystery of divisibility by 11 remained unsolved unless the actual division was performed. We suggest that these students may not "believe," or at least not "believe in practice," in the Fundamental Theorem of Arithmetic that assures the uniqueness of prime decomposition. Another possible explanation for this phenomenon is that the conceptual understanding of divisibility and indivisibility have not developed at the same rate. It may be the case that for Patty divisibility has been conceptualized whereas indivisibility has not.

In the second part of Question Set 2-"Is 391 divisible by 46 ?"-there was no unanimous conclusion among the 17 participants who were asked this question. This part of the question was presented only after participants had answered the first part; that is, had determined the divisibility of 391 by 23 and expressed 391 as $23 \times 17$, by whatever means.

When presented with the second part of Question Set 2, four participants immediately used a calculator and based their conclusion on the calculator's result. After concluding that 391 was not divisible by 46 with the help of her calculator, Armin was invited to think of another strategy:

Interviewer: My question is: if you didn't have your calculator with you, how would you think about this? 391 , is it divisible by 46 ? What would you do?

Armin: (Pause) Um, I guess I'd just have to guess out of the blue, I would say, no, but, I mean, I would never trust my own opinion, I always have to work it out just to see (laugh).

Because Armin lacked any viable alternative to "guess(ing) out of the blue," it seems that carrying out the calculation was her only recourse to addressing this problem.

However, attempts to use more advanced mathematical reasoning did not necessarily lead to correct conclusions. On the same question, 5 out of 17 participants claimed that 391 was indeed divisible by 46 , because " 46 is just 23 doubled." Anita was tempted toward this way of thinking and yet was able to correct herself with the use of a specific numerical example: 
Interviewer: Okay, right. Um, would 391 be divisible by 46 ?

Anita: Yes.

Interviewer: And why so?

Anita: Oh, maybe not.

Intervicwer: $\quad \mathrm{I} \mathrm{m}, \mathrm{I} \mathrm{m}$ interested in both of those things that just happened to you. I'm interested in the "yes" and the "maybe not."

Anita: Well, first I said yes because I thought 46 is, well 23 is a factor, is a factor of 46 . it's $23 \times 2$, um, but then again, I thought the 5 is a factor, like, for example, 5 is a factor of 25 but 10 isn't, and so just because it's doubled doesn't mean it's a factor of, so I'm not too sure?? I think I'd have to say no.

Bob, Patty, and Anabelle demonstrated in their answers various levels or degrees of sophistication. The arguments presented in the following excerpts progress from considering the possible last digit in a multiple of 46 , to considering the "evenness" of number 46.

Interviewer: Okay. How about 46 , would 391 be divisible by 46 ?

Bob: (pause) No, it wouldn't because uh in 46 the unit digit is 6 . and the units digit of 391 is 1 , and 6 . knowing the multiples of 6 , I know that there will not be a units digit of 1 after being multiplied by 6 . For example, $6 \times 6$ is 36. units digit and that is obviously 6 .

Based on considering the last digit for the multiples of 6 , Bob realized that no multiple of 6 will end with 1 . Here Bob had constructed a novel procedural understanding of indivisibility of 391 by 46 . Patty makes a further step when she, similarly to Bob, considers the last digit, but also considers the events and oddness of the numbers in question.

Patty: $\quad$ Because 46 ends with an even number and 391 is an odd number. .

Interviewer: $\mathrm{Um} \mathrm{hm}$.

Patty: And 6 is even. it won't fit into an odd number.

We note that in Patty's response, "it won't fit into," the procedural aspect of division is emphasized. That Anabelle's explanation is yet further refined is evidenced in her consideration of 46 itself as an even number, and not just considering its even last digit.

Interviewer: Okay. Alright. Now l'd like to ask you if 391 would be divisible by 46 ? Anabelle: No, because it [46] was an even number. and this one [391] is an odd number.

Arguments related to odd and even numbers were repeated by 5 participants. However, it is not clear from our data how generalizable those arguments are. 
Our investigations in this area have revealed that encapsulation of divisibility by 2 (i.e., the conceptualization of the evenness or oddness of a number) need not occur simultaneously with encapsulation of divisibility by $3,4,5$, or any other number (Zazkis \& Campbell, 1994). It is not evident that Anabelle's understanding of divisibility is general enough to accommodate other cases. For instance, if we had asked whether or not 391 was divisible by $69(3 \times 23)$, Anabelle's reasoning here would no longer have been applicable and she may have had to resort to calculation. Further research is needed to determine the extent of generalizability of arguments based on this odd-even heuristic.

Explicit awareness of factors of 46 and the argument that 391 could not be divisible by 46 , because it has a factor 2 that is not one of the factors of 391 , was given by only one participant, Dana:

Interviewer: Okay, um, would you say 391 is divisible by 46 ?

Dana: (Pause) No, because 23 and 17 are both prime numbers, there is no 2 involved in there, it's just 23 times 17.

Dana has made a powerful connection between the concepts of divisibility and prime decomposition to refute divisibility of 391 by 46 . In her words, "there is no 2 involved in there," we recognize a hint to the uniqueness of prime decomposition of 391. It is evident that an understanding of the uniqueness of prime decomposition lends itself much more readily to generalized forms of arguments regarding divisibility.

We also found in the interview data some explicit beliefs regarding the nature of prime decomposition. A belief that was evidenced explicitly in four interviews is that decomposition into prime factors means decomposition into small prime factors. This belief is demonstrated in the following excerpts with Lisa and Tanya:

Interviewer: Um, let's take the number 391 . Would 391 be divisible by 23 ?

Lisa: 23, (pause), I don't know. I don't think so.

Interviewer: $\mathrm{Hmm}$, and why do you think not?

1. . . .

Lisa: 391 , I think, is a prime.

Interviewer: And why do you think that 391 is a prime?

Lisa: Interviewer: Because I don't think it had been divided by 2 or 3 , or 5 , or 7 (laugh) . . (laugh)

Lisa:

Like I'm going through my mind with primes, and I know that it's not divisible by 2 because it's not an even number. I know it's not divisible by 3 , because the sum isn't um divisible by 3 . Four, same reason for the 2 , it's not even. Five, it has to be a 5 or a 0 , um, 7 , it's not divisible by 7 .

Interviewer: And, what would be the next prime?

Lisa: $\quad 11$.

Interviewer: You think it's divisible by 11 ? 


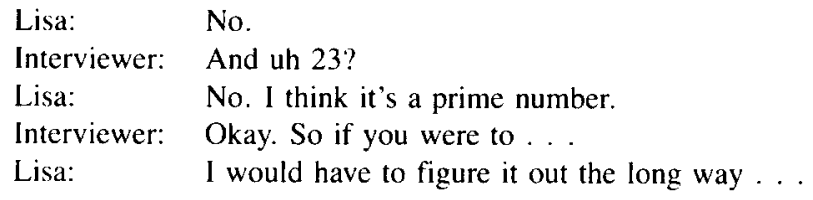

"The long way" for Lisa here means to keep dividing by primes. It is interesting to note that she stopped at 11 to infer the primeness of 391 , and did not even try to divide by 23 , in order to obtain a direct answer for the interview question. Tanya, as well as Lisa, conjectured that 391 was a prime number. To the interviewer's request for justification, she replied:

Tanya: I don't know. I guess, like I, um, like I was saying with, I know there's a way to do it, prime factorization, and I know that 23 is a prime number, but I guess, um, I was assuming, for some reason, that as long as 391 was not a prime number, it would have a factor smaller than 23 , a prime factor smaller than 23.

Interviewer: And is there a reason why, why you thought that way'?

Tanya: Um, I guess because in, in my experience in most cases, a large number, relatively large number like 391 , would have, well any number not even a large number, any number has um some small prime factors in addition to whatever else we have, we may have a large number, prime factors like 23 , but they also tend to have things like 2 and 3 and 5 and 7 .

Interviewer: Well what if we took 2 very large prime numbers?

Tanya: $\quad \mathrm{Um} \mathrm{hm} \ldots$

Interviewer: And multiplied them together to get another number?

Tanya: Um hm.

Intervicwer: Would that number have a small prime in its prime factorization?

Tanya: (Pause) Umm, no, I don't think so.

|. . . .

Tanya: I guess it's probably just more experience than anything, but it just seems to me that when you factor a number into its primes, I mean what you're doing is, you're trying to find the smallest, I mean numbers that can no longer be broken into anything smaller aside from 1 and itself, so that. I guess it's just the whole idea of factoring things down into their smallest parts ...

Interviewer: Um hm.

Tanya: I guess gives me the idea that those parts are themselves going to be small.

It is interesting to note here, that Tanya's belief that prime decomposition is decomposition into "small" primes coexists with her awareness of existence of very large primes.

\section{DISCUSSION}

Sfard (1992) pointed out the difficulty in reification in general and suggested that, at some particular point or level, it may be "out of reach" for some learners. 
We have illustrated this difficulty in the case of prime decomposition of a composite number into its prime factors. It may be the case that this difficulty stems from students' previous experiences of expressing composite numbers as products. The number 96 , for example, can be written as $16 \times 6$ or $8 \times 12$. Obviously, the decomposition is not unique. Uniqueness, independent of order, appears only when the number has been completely decomposed into factors that are, one and all, prime. This seems to be the major difference, between factors and prime factors, that needs further pedagogical attention. The concept of prime decomposition is a unifying schema for prime and composite numbers; that is, it relies on these concepts and their interrelation. If the concepts of prime and composite numbers have not been adequately constructed, this will likely inhibit any meaningful conceptualization of prime decomposition. Although we have yet to empirically determine this to be the case, we have investigated similar phenomena with respect to inadequate encapsulations of conceptual objects, such as distributivity, factoring, and multiplication, used in higher order processes pertaining to divisibility (Campbell \& Zazkis, 1994; Zazkis \& Campbell, 1996).

We hypothesize that the concept of divisibility is usually constructed, or encapsulated as an object, prior to its negative counterpart, the concept of indivisibility. This is supported by the success of 16 participants to recognize 3,5 , and even 15, among the divisors of $M$ (Question 1) followed by a failure to recognize 11 among $M$ 's nondivisors. We note here that although the two inferences, " 7 is one of $M$ 's prime factors, therefore $M$ is divisible by 7 ," and " 11 is not one of $M$ 's prime factors, therefore $M$ is not divisible by 11 ," share similar linguistic structure, they are neither logically nor conceptually equivalent. In particular, the latter requires a conceptual understanding of the uniqueness of prime decomposition, whereas the former does not. We also find evidence suggesting that many students believe that decomposition into prime components means decomposition into small prime components, a belief that is quite likely based on extensive examples using small numbers from prior school experiences and textbook examples.

We belicve that a proper understanding of the concept of prime decomposition is central for the understanding of the structure of whole numbers. However, our data demonstrate that the Fundamental Theorem of Arithmetic has not been adequately grasped by a large number of preservice elementary school teachers. Whereas the existence of prime decomposition may be taken for granted, the uniqueness of prime decomposition appears to be counterintuitive and often a possibility of different prime decompositions is assumed. There is also a possibility, consistent with findings of other researchers (Fischbein \& Kedem, 1982; Martin \& Harel, 1989; Schoenfeld, 1988), that our participants do not understand the meaning and significance of the concept of theorem. Because the proof for the Fundamental Theorem of Arithmetic is most likely to be omitted in basic mathematics courses for preservice elementary school teachers, we feel that some pedagogical alternative is needed as compensation. In particular, we sug- 
gest that specific instructional techniques based on the types of assessment questions explored herein offer a means for preservice teachers to understand the procedural and conceptual dimensions of prime decomposition and the Fundamental Theorem of Arithmetic.

\section{REFERENCES}

Artigue, Michelle (1992, August). Tool and object status of mathematical concepts: The case of complex numbers. Paper presented at the Advanced Mathematical Thinking Working Group, PME XVI, Durham, NH.

Campbell, Stephen, \& Zazkis, Rina (1994). The distributive flaws: Latent conceptual gaps in preservice teachers' understanding of the property relating multiplication to addition. In D. Kirshner (Ed.), Proceedings of the Sixteenth Annual Meeting of the North American Chapter of the International Group for the Psychology of Mathematics Education (Vol. 2, pp. 268-274). Baton Rouge, LA: PME.

Douady, Regina (1985). The interplay between different settings: Tool-object dialectic in the extension of mathematical ability - Examples from elementary school teaching. In L. Streefland (Ed.), Proceedings of the Ninth International Conference of PME (Vol. 2, pp. 33-52). Utrecht, The Netherlands: PME.

Dubinsky, Ed (1991). Reflective abstraction in advanced mathematical thinking. In D. Tall (Ed.), Advanced mathematical thinking (pp. 95-126). Boston: Kluwer Academic.

Dubinsky, Ed, Leron, Uri, Dautermann, Jennie, \& Zazkis, Rina (1994). On learning fundamental concepts of group thcory. Educational Studies in Mathematics, 27(3), 267-305.

Fischbein, Efraim, \& Kedem, Irit (1982). Proof and certitude in the development of mathematical thinking. In A. Vermandel (Ed.), Proceedings of the Sixth International Conference for the Psychology of Mathematics Education (pp. 128-131). Antwerp, Belgium: PME.

Freudenthal, Hans (1983). The didactical phenomenology of mathematical structures. Dordrecht, The Netherlands: Reidel.

Greeno, James G. (1983). Conceptual entites. In D. Gentner \& A.L. Stevens (Eds.), Mental models (pp. 227-252). Hillsdale, NJ: Erlbaum.

Harel, Guershon, \& Kaput, Jim (1991). The role of conceptual entities and their symbols in building advanced mathematical concepts. In D. Tall (Ed.), Advanced mathematical thinking (pp. 8294). Boston: Kluwer Academic.

Martin, Gary, \& Harel, Guershon (1989). Proof frames of preservice elementary teachers. Journal for Research in Mathematics Education, 20(1), 41-51.

Piaget, Jean (1972). The principles of genetic epistemology (W. Mays, Trans.). New York: Basic Buoks.

Schoenfeld, Alan (1988). When good teaching leads to bad results. Educational Psychologist, 23(2), 145-166.

Sfard, Anna (1991). On the dual nature of mathematical conceptions: Reflections on processes and objects as different sides of the same coin. Educational Studies in Mathematics, 22, 1-36.

Sfard, Anna (1992, August). On operational-structural duality of mathematical conceptions. Paper presented at Advanced Mathematical Thinking Working Group, PME XVI, Durham. NH.

Zazkis, Rina, \& Campbell, Stephen (1994). Divisibility and division: Procedural altachments and conceptual understanding. In J. Matos (Ed.). Proceedings of the 18th International Conference for Psychology and Mathematics Education (Vol. 4, pp. 423-430). Lisbon, Portugal: PME.

Zazkis, Rina, \& Campbell. Stephen (1996). Divisibility and multiplicative structure of natural numbers: Preservice teachers' understanding. Journal for Research in Mathematics Education, 27(5). 